\title{
Polarization-Insensitive Circular Reflectarray for Satellite Applications in Ka-band
}

\author{
Ali Jihad Ali, Mohsen Khalily, Ali Araghi, Rahim Tafazolli \\ 5G \& 6G Innovation Centres (5GIC \& 6GIC), Institute for Communication Systems (ICS) \\ University of Surrey, Guildford, UK \\ \{ali.ali,m.khalily\}@surrey.ac.uk
}

\begin{abstract}
A polarization-insensitive circular reflectarray antenna (RA) for long-distance wireless communications is investigated. By combining patches, dipoles, and rings, a polarizationinsensitive unit cell is achieved. With a phase variation of around $314^{\circ}$ between $30 \mathrm{GHz}$ and $32 \mathrm{GHz}$, a circular reflectarray with a radius of $400 \mathrm{~mm}$ is built. Simulation results indicate a maximum realized gain of $27.6 \mathrm{~dB}$ at $30 \mathrm{GHz}$.
\end{abstract}

\section{INTRODUCTION}

The interest in planar antennas has significantly raised to reduce the cost and volume occupied by the RF systems. Especially for satellite applications since the available surface and volume are limited. Hence, the antennas for space missions must be as small as possible in terms of the weight, size, and volume as in the case of reflectarray antennas (RAs) [1]. Printed RAs show several advantages for space applications compared to conventional antennas in terms of weight and cost, as well as the ease of fabrication. More importantly, RAs offer a higher level of flexibility to regulate the radiation characteristics such as the direction or polarization of the reflected beam(s) [2].

RAs utilize arrays of sub-wavelength printed patches in a periodic form to reflect the incident waves in different directions based on the elements arrangement [3]. In periodic structures [4], the smallest element that is repeated in a fashion is called a unit cell. The shape of the unit cell plays a significant role in the performance of RAs. Although these structures offer high gain in the microwave and $\mathrm{THz}$ ranges, the main limitation of RAs is the limited bandwidth. Several solutions are introduced to broaden the narrow bandwidth of RAs as designing stacked patches of variable size, aperture coupled elements, and multi-resonant unit cells on a singlelayer [2]. In pursuit of achieving a bandwidth of $2 \mathrm{GHz}$, a multi-resonant unit cell is chosen in this paper. Most of the RAs in the literature introduced unit cells that suffer from polarization sensitivity. The proposed unit cell offers an almost invariant EM-characteristics for different polarizations, this can guarantee a homogeneous response of the RA against different polarizations of the feeder.

\section{Antenna CONFIGURATION AND DESIGN}

Conventionally, the main parts of RAs are a feed horn and a reflecting EM-aperture embodied by several unit cells. Fig. 1 shows the proposed unit cell and the layout of the corresponding EM-aperture. In this paper, a conical horn

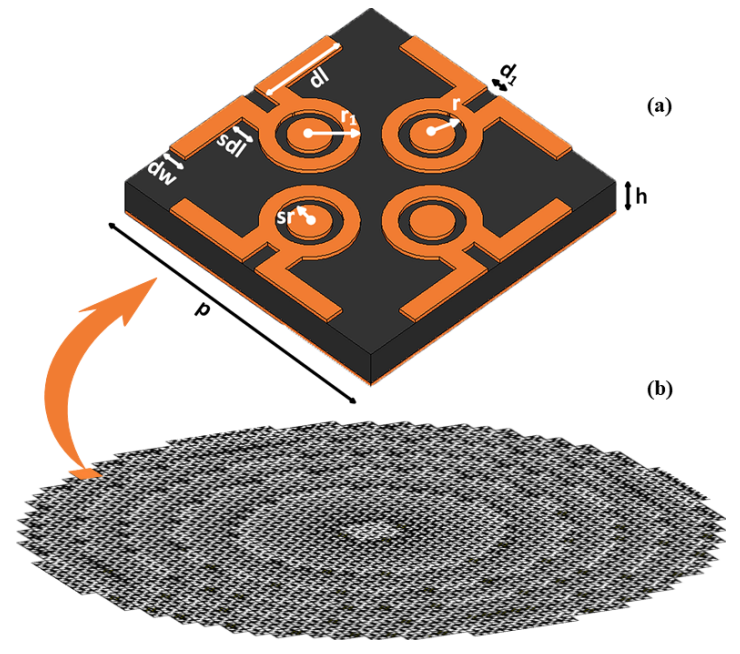

Fig. 1. Sketch of the antenna. (a) unit cell (b) reflectarray.

antenna with a realized gain of $18.6 \mathrm{~dB}$ at $30 \mathrm{GHz}$ is utilized as the feeder.

\section{A. Proposed Unit Cell}

The proposed unit cell is constituted of four patches, four rings, and dipoles of copper. It is printed on one side of Rogers TMM3 dielectric substrate with a thickness of $h=0.8$ $\mathrm{mm}$. The dimensions and geometries of the proposed element are as follows: $r w=0.5 \times r, r_{1}=r+r w, p=4.7$ $\mathrm{mm}, s r=0.5 \times r_{1}, d_{1}=0.5 \times r, s d l=0.83-0.1 \times r$, $d w=0.65 \times r$, and $d l=3 \times r$, where $r$ varies from $0.26 \mathrm{~mm}$ to $0.54 \mathrm{~mm}$. According to these values of $r$, Fig. 2 displays the simulated phase shift of reflection at the frequency range of $f=30-32 \mathrm{GHz}$. As observed from this figure, a smooth phase transition over the specified bandwidth is achieved. For instance, a phase-shift of about $321^{\circ}$ at $30 \mathrm{GHz}$ is obtained, which is sufficient enough to generate the desired beam as it is above $300^{\circ}$ [5]. In addition, the reflection amplitude is less than $-0.8 \mathrm{~dB}$ throughout the entire range of study. That guarantees a proper response out of the whole aperture while the unit cell dimensions vary. Results are obtained by CST Studio Suite with the periodic boundary condition and Floquet excitation.

As for the element arrangement, a unit cell located at a certain point of the reflectarray ought to impart the following 


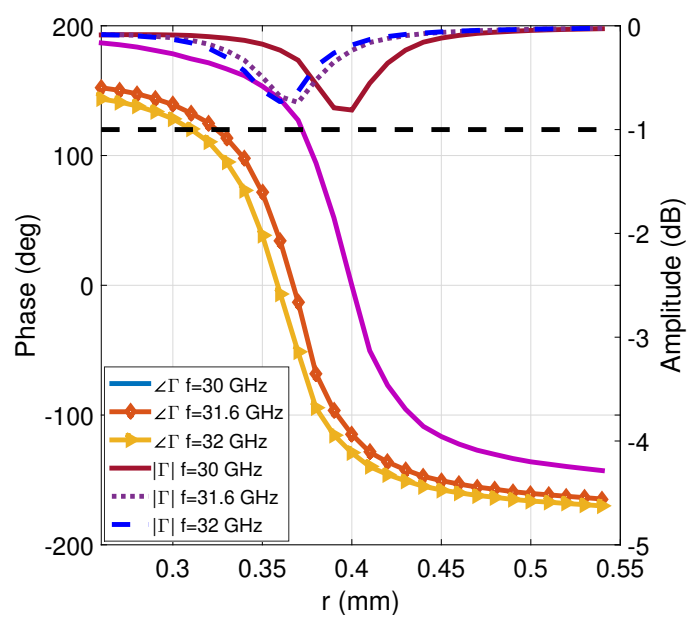

Fig. 2. Phase and amplitude responses of the element.

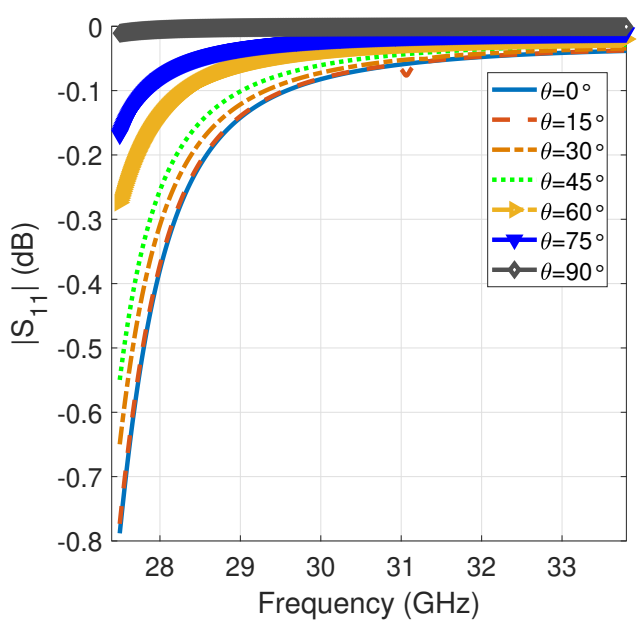

Fig. 3. Reflection coefficient of the unit cell for different incident angles.

phase to the incoming spherical field that originated from the focal point (opposite to the incident wave direction) [6]:

$$
k_{0}\left(F A-\overrightarrow{F O} \cdot \hat{r}_{\text {out }}\right)-\phi_{A}=2 n \pi
$$

where $k_{0}$ is the free space wave-number, $O$ is the center of the reflecting sheet, $\hat{r}_{\text {out }}$ is the direction of the outgoing beam, and $n$ is an integer. As for $\phi_{A}$, it is the scattered phase generated by the patch located at point "A".

\section{B. Simulation Results}

Considering different transversal angles $(\theta)$ of impinging waves on the unit cell, Fig. 3 shows that the proposed unit cell is not highly sensitive to the polarization of the incident waves, particularly at the desired frequency range of $f=30-32 \mathrm{GHz}$. This happens because of the combination of rings, patches, and dipoles in the geometry of the introduced unit cell.

The reflector with a radius of $400 \mathrm{~mm}$ is formed via several size-modulated printed patches. The required phase distribution pattern on the reflector is obtained by Eq. (1).

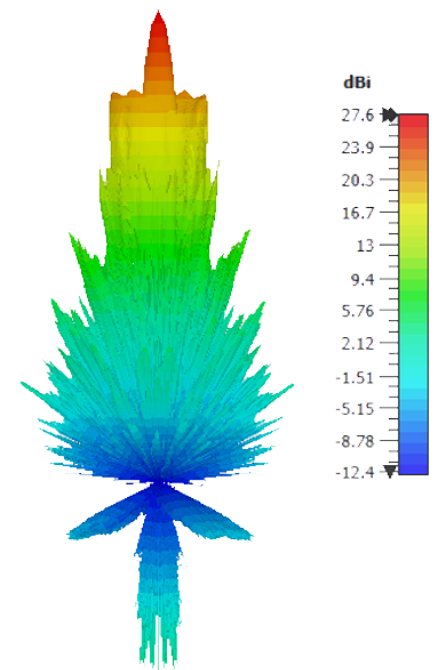

Fig. 4. The 3D far-field radiation pattern at $30 \mathrm{GHz}$.

Based on this phase distribution, the modulation procedure is regulated by the unit cell response (e.g. Fig. 2). That makes the aperture properly sample the phase pattern so that the incoming waves can be effectively redirected to the direction of interest [7]. The constructed radiation pattern is shown in Fig. 4, stating that the proposed RA offers $27.6 \mathrm{dBi}$ gain at $30 \mathrm{GHz}$, which is high enough for LEO-based satellite communications [8].

\section{CONCLUSION}

This work presented a circular RA with a novel unit cell for space applications. A polarization-insensitive aperture in the Ka-band was developed for this purpose with an operational bandwidth from $30 \mathrm{GHz}$ to $32 \mathrm{GHz}$.

\section{REFERENCES}

[1] Volkan Akan and Erdem Yazgan (August 18th 2020). Antennas for Space Applications: A Review, Advanced Radio Frequency Antennas for Modern Communication and Medical Systems, Albert Sabban, IntechOpen, DOI: 10.5772/intechopen.93116.

[2] R. Florencio, J. A. Encinar, R. R. Boix, V. Losada and G. Toso, "Reflectarray Antennas for Dual Polarization and Broadband Telecom Satellite Applications," in IEEE Transactions on Antennas and Propagation, vol. 63, no. 4, pp. 1234-1246, April 2015, doi:10.1109/TAP.2015.2391279.

[3] D. Berry, R. Malech and W. Kennedy, "The reflectarray antenna," in IEEE Transactions on Antennas and Propagation, vol. 11, no. 6, pp. 645-651, November 1963, doi: 10.1109/TAP.1963.1138112.

[4] A. Araghi, M. Khalily, P. Xiao, R. Tafazolli, "Holographic-based mmwwideband bidirectional frequency scanning leaky wave antenna," in 14th European Conference on Antennas and Propagation (EuCAP), 2020.

[5] Nayeri, P., Yang, F., \& Elsherbeni, A. (2018). Reflectarray Antennas: Theory, Designs, and Applications Reflectarray Antennas: Theory, Designs, and Applications.

[6] Shaker, Jafar, et al. Reflectarray Antennas: Analysis, Design, Fabrication, and Measurement. Artech House, 2014.

[7] M. H. Dahri, M. H. Jamaluddin, M. Khalily, M. I. Abbasi, R. Selvaraju and M. R. Kamarudin, "Polarization Diversity and Adaptive Beamsteering for 5G Reflectarrays: A Review," in IEEE Access, vol. 6, pp. 1945119464, 2018, doi: 10.1109/ACCESS.2018.2821358.

[8] S. Anguix, A. Araghi, M. Khalily, R. Tafazolli, "Reflectarray Antenna Design for LEO Satellite Communications in Ka-band," in 15th European Conference on Antennas and Propagation (EuCAP), 2021. 